\title{
Overlapping cleavage motif selectivity of caspases: implications for analysis of apoptotic pathways
}

\author{
GP McStay ${ }^{1}$, GS Salvesen ${ }^{2}$ and DR Green ${ }^{*, 1}$
}

\begin{abstract}
Caspases orchestrate the controlled demise of a cell after an apoptotic signal through specific protease activity and cleavage of many substrates altering protein function and ensuring apoptosis proceeds efficiently. Comparing a variety of substrates of each apoptotic caspase $(2,3,6,7,8,9$ and 10$)$ showed that the cleavage sites had a general motif, sometimes specific for one caspase, but other times specific for several caspases. Using commercially available short peptide-based substrates and inhibitors the promiscuity for different cleavage motifs was indicated, with caspase-3 able to cleave most substrates more efficiently than those caspases to which the substrates are reportedly specific. In a cell-free system, immunodepletion of caspases before or after cytochrome $c$-dependent activation of the apoptosome indicated that the majority of activity on synthetic substrates was dependent on caspase-3, with minor roles played by caspases- 6 and -7. Putative inhibitors of individual caspases were able to abolish all cytochrome $c$-induced caspase activity in a cell-free system and inhibit apoptosis in whole cells through the extrinsic and intrinsic pathways, raising issues regarding the use of such inhibitors to define relevant caspases and pathways. Finally, caspase activity in cells lacking caspase-9 displayed substrate cleavage activity of a putative caspase-9-specific substrate underlining the lack of selectivity of peptide-based substrates and inhibitors of caspases.
\end{abstract}

Cell Death and Differentiation (2008) 15, 322-331; doi:10.1038/sj.cdd.4402260; published online 2 November 2007

Execution of apoptosis depends on the function of caspase proteases. Activation of these proteases can occur in two ways, through induced oligomerization of the monomeric initiator caspases (2, 8, 9 and 10), or through cleavage of the dimeric executioner caspases $(3,6$ and 7$) .{ }^{1}$ The induced proteolytic activity of a caspase acts on a series of specific substrates that ensures the apoptotic program progresses efficiently. ${ }^{2}$ The general schemes of apoptotic caspase cascades are known and are divided into two pathways. The intrinsic pathway is mediated through mitochondrial outer membrane permeabilization resulting in cytochrome $c$ release. The released cytochrome $c$ activates apoptotic protease activating factor (APAF-1) in concert with adenine trinucleotides to form the apoptosome by recruiting and activating caspase- $-9{ }^{3}$ The apoptosome then activates caspase- 3 and -7 through direct cleavage by caspase- 9 . The extrinsic pathway is engaged by ligating death receptors of the tumor necrosis factor receptor family. Through engagement of adapter molecules, caspase- 8 and -10 are recruited to the death-induced signaling complex $\left(\mathrm{DISC}^{4,5}\right)$. Activated caspase- 8 can then process BID leading to permeabilization of the mitochondrial outer membrane, leading to cytochrome $c$ release ${ }^{6,7}$ and activation of the apoptosome akin to the intrinsic apoptotic pathway. In addition, caspase-8, in some cell types, is sufficient to cleave caspase-3 directly, ${ }^{8}$ bypassing the need for mitochondrial outer membrane permeabilization, and resulting in apoptosis.

One strategy to determine a caspase's role in apoptotic cascades uses a series of peptide-based substrates and inhibitors developed as specific probes for caspase activity based on the favored substrates of the caspases. ${ }^{9,10}$ The cleavage specificities for each caspase indicated that several amino acids could be tolerated in each position of the cleavage motif and rarely were single amino acids crucial for specificity at any given position, apart from the defining aspartate residue located $\mathrm{N}$-terminally adjacent to the cleavage site. A study determining the specificity of peptidebased inhibitors using a variety of sequences revealed that some of the inhibitors were able to inhibit more than one caspase, with little selectivity. ${ }^{11}$ These peptide-based substrates and inhibitors have been used extensively to monitor caspase dependence of cell death and to identify caspases involved in different scenarios of apoptosis. However, problems of specificity have recently been reported for these reagents. ${ }^{2,12,13}$ Because of this, using peptide-based substrates and inhibitors may lead to flawed conclusions as peptide-based tools may not target a single caspase in an environment where multiple caspases exist. Studies based on these tools rarely consider other targets of the substrate or inhibitor used, perhaps leading to discrepancies between

\footnotetext{
${ }^{1}$ Department of Immunology, St. Jude Children's Research Hospital, 332 North Lauderdale Street, Memphis, TN 38105, USA and ${ }^{2}$ Apoptosis \& Cell Death Research Program, The Burnham Institute for Medical Research, 10901 North Torrey Pines Road, La Jolla, CA 92037, USA

*Corresponding author: DR Green, Department of Immunology, MS 351, St. Jude Children's Research Hospital, 332 North Lauderdale Street, Memphis, TN 38105 USA. Tel: + 1901 4953470; Fax: + 1901 4955766; E-mail: Douglas.green@ stjude.org

Keywords: caspase; substrate; inhibitor; apoptosis; selectivity

Abbreviations: APAF-1, apoptotic protease activating factor-1; BID, BH3 interacting death agonist; CARD, caspases recruitment domain; dATP, deoxyadenosine triphosphate; DED, death effector domain; DISC, death-induced signaling complex; DMSO, dimethyl sulfoxide; DTT, dithiothreitol; FBS, fetal bovine serum; FITC, fluorscein isothiocyanate; HEPES, 4-(2-hydroxyethyl)piperazine-1-ethanesulfonic acid; MOMP, mitochondrial outer membrane permeabilization; RPMI, Roswell Park Memorial Institute; UV, ultraviolet

Received 12.4.07; revised 24.9.07; accepted 24.9.07; Edited by G Melino; published online 02.11.07
} 
conclusions drawn from studies using these and other methods.

Our goal was to examine the selectivity of allegedly specific caspase substrates and inhibitors by assaying activity of recombinant caspases, in vitro activation of caspases and induction of apoptosis in cells. Our data show that the substrates and inhibitors are not specific for a particular caspase and that caspase- 3 is the most efficient target of the majority of these peptide motifs. We have found that caspase3 has the most prevalent activity induced by the mitochondrial pathway of apoptosis, with minor roles played by caspase- 6 and -7 , and these three caspases account for the majority of activity regardless of the caspase being allegedly monitored.

\section{Results}

Comparisons of caspase cleavage motifs. To reassess caspase cleavage motifs in proteins reported to act as substrates for caspases,,$^{2,3,6-10}$ we analyzed the human protein reference database (www.hprd.org). This database compiles lists of protease cleavage sites, and with the caveat that it is biased by what can be found in the literature, it is a suitable source for understanding the specificity on protein substrates. The amino acids making up the motif upstream of the cleavage site $(P 4,3,2,1)$ and the amino acid after the cleavage site $\left(P 1^{\prime}\right)$ were counted for all the substrates available on the database where the cleavage motif could be readily discerned. ${ }^{14}$ The presence of amino acids in cleavage motifs was weighted equally, although different systems were employed to identify these, and therefore caution should be used in interpreting these results. The amino acids from each position were counted and displayed as a percentage of total cleavage motifs available. The frequency of amino acids at each position in cleavage motifs was consistent for most of the caspases. The most prominent motif was the Glu-Val-Asp amino-acid sequence at P3, P2 and P1 (Supplementary Figure 1). This motif was common for caspase-3, -6, -7, -8, -9 and -10 (for caspase-2 a prevalence for amino acids in the cleavage motif could not be determined from the three substrates provided by the database). Caspase- 3 and -7 were the only members of the family to display the cleavage motif predicted by Thornberry et al., ${ }^{10}$ Asp-Glu-Val-Asp. The caspase-8 motif (Leu-Glu-Thr-Asp) differs from the predicted motif used in the following studies by only one amino acid
(lle-Glu-Thr-Asp). The difference at P4 is with amino acids with similar physico-chemical properties. The most prevalent caspase- 9 cleavage motif, Ser-Glu-Val-Asp, differed dramatically from the motif used in the following experiments, Leu-Glu-His-Asp. The amino acid at P2 being different from each other with respect to physicochemical properties, valine, a small hydrophobic amino acid compared to histidine, a charged bulky amino acid. The amino acid after the cleavage site usually was a small amino acid, most commonly glycine, followed by threonine or serine, consistent with what has been found for small synthetic substrates. ${ }^{15}$ These comparisons demonstrated that caspases share similar cleavage motifs in their physiological substrates. These observations prompted us to determine the specificity of the commercially available tetrapeptide motif-based tools for analysis of caspase activity.

Activity of recombinant caspases. To determine the activity of caspases against different substrates an equimolar amount of caspase-active sites, determined by zVAD-FMK titration, was used (Table 1). Truncated versions of the initiator caspases were used as this renders them much easier to purify with cleavage activity similar to that found with full-length caspases. ${ }^{1,8,16}$ These titrations would also ensure that truncated initiator caspases were used at an equal amount determined by activity, rather than total protein, compared to the executioner caspases. By using equimolar activities of recombinant caspases, comparisons of the relative efficiencies of cleavage for the different caspases and substrates could be made with confidence that equal amounts of activity were used, thus discounting inactive enzyme that may be present in preparations of recombinant caspase.

Consistent with another report, ${ }^{9}$ caspase-2 cleaved the motif VDVAD most efficiently and showed little activity against other substrates. Caspase-3 cleaved DEVD most efficiently, but cleaved other substrates well. Similarly, caspase-6 cleaved VEID most efficiently as reported ${ }^{9}$ but also demonstrated efficient cleavage of IETD and LEHD. Caspase-7 had a similar profile to caspase-3, cleaving DEVD most efficiently. Caspase-8 showed greater cleavage efficiency for the substrate LEHD, a putative marker for caspase- 9 activity, ${ }^{10}$ rather than its putative specific substrate IETD. Caspase- 9 itself showed greatest specificity for its intended substrate

Table 1 Cleavage of caspase substrates by recombinant caspases

\begin{tabular}{|c|c|c|c|c|c|}
\hline \multirow[b]{3}{*}{ Caspase } & \multicolumn{5}{|c|}{ Substrate (AFC) } \\
\hline & VDVAD & DEVD & VEID & IETD & LEHD \\
\hline & \multicolumn{5}{|c|}{ Substrate cleavage (mean RFLU change/min/100nM caspase active site \pm S.D.) } \\
\hline 2 & $2.20 \pm 0.54$ & $0.04 \pm 0.01$ & $-0.01 \pm 0.01$ & $0.02 \pm 0.01$ & $0.17 \pm 0.07$ \\
\hline 3 & $10.20 \pm 1.36$ & $24.04 \pm 3.89$ & $5.88 \pm 0.95$ & $2.98 \pm 0.52$ & $2.74 \pm 0.64$ \\
\hline 6 & $0.10 \pm 0.02$ & $0.97 \pm 0.05$ & $3.74 \pm 0.18$ & $2.93 \pm 0.19$ & $2.33 \pm 0.29$ \\
\hline 7 & $1.17 \pm 0.21$ & $4.91 \pm 0.69$ & $0.12 \pm 0.03$ & $0.06 \pm 0.01$ & $0.06 \pm 0.02$ \\
\hline 8 & $0.08 \pm 0.01$ & $0.82 \pm 0.03$ & $0.48 \pm 0.02$ & $1.83 \pm 0.05$ & $3.26 \pm 0.24$ \\
\hline 9 & $0.01 \pm 0.01$ & $0.03 \pm 0.01$ & $0.05 \pm 0.01$ & $0.11 \pm 0.03$ & $1.08 \pm 0.37$ \\
\hline 10 & $0.21 \pm 0.13$ & $0.60 \pm 0.36$ & $0.75 \pm 0.38$ & $1.48 \pm 0.82$ & $1.88 \pm 1.06$ \\
\hline
\end{tabular}


LEHD, but not other substrates. Caspase-10a showed a similar pattern to caspase-8, preferring the caspase-9 substrate LEHD rather than its putative substrate IETD. These results confirm that some caspases can cleave some substrates better than others, and sometimes those not expected based on previous analyses. ${ }^{9,10}$ These data show that for each substrate, in most cases, caspase- 3 shows the most efficient cleavage activity (Table 1), while having a similar activity to caspase- 6 for the IETD substrate and caspase- 8 for the LEHD substrate. Analysis of the results in this way demonstrated that caspase- 3 is the most promiscuous caspase tested, cleaving substrates better than most of the caspases for which the substrate was designed, thereby confirming prior conclusions regarding caspase-3 activity on multiple supposedly caspase-selective substrates. ${ }^{15}$

Caspase activity induced by cytochrome $\boldsymbol{c}$ activation of the apoptosome. To extend this analysis to physiologically activated caspases, we used a well-described system of caspase activation by adding cytochrome $c$ to cytosolic extracts. ${ }^{3,17}$ Cytochrome $c$ induces oligomerization of APAF-1 in the presence of adenine trinucleotides that acts as a platform for the dimerization and activation of procaspase- 9 leading to cleavage and activation caspase- 3 and $-7,{ }^{1,16}$ which in turn cleave and activate caspase- $6 .{ }^{18}$ Caspase activities induced by cytochrome $c$ in a dosedependent manner were determined in cytosolic extracts in the presence of $1 \mathrm{mM} d A T P$. Concentrations greater than $400 \mathrm{nM}$ cytochrome $c$ induced complete caspase activity for all substrates tested (Figure 1a). The caspase-3/-7 substrate DEVD was the most readily cleaved substrate in terms of total activity and fold induction. Cleavage of the caspase- 6 substrate VEID and the caspase- 9 substrate LEHD were also induced. Interestingly the other caspase substrates tested VDVAD, a caspase-2 substrate, and IETD, a caspase-8/-10 substrate were significantly cleaved, with VDVAD being the second most cleaved substrate, greater than substrates predicted to be cleaved in this pathway, VEID and LEHD.

To examine the role of each caspase a series of immunodepletion experiments were performed. Antibodies to caspase-2, $-3,-6,-7,-8$ and -9 removed all detectable fulllength caspase from cytosolic extracts as detected by Western blotting (Figure 1b and Supplementary Figure 2a). Using the immunodepleted extracts, caspase activation was achieved by adding cytochrome $c$ and dATP followed by substrate cleavage assays (Table 2 and Supplementary Figure $2 \mathrm{~b}$ ). In non-depleted extracts and control immunodepletions using mouse or rabbit immunoglobulin, full substrate cleavage activity was observed upon addition of cytochrome $c$ and dATP. As predicted, ${ }^{18,19}$ immunodepletion of procaspase-9 from the cytosolic extract rendered the extracts resistant to cytochrome $c$-induced cleavage of any caspase substrate confirming that each activity was due to an apoptosome-mediated caspase-9-dependent cascade. Interestingly, the caspase-3 immunodepleted extract gave consistently decreased amounts of caspase activity prior to the addition of cytochrome $c$ and dATP. Caspase-3-depleted cytosolic extracts lost all VEID, IETD and LEHD cleavage activity, and only partial cleavage of VDVAD and DEVD was observed. Therefore these cleavage activities induced by

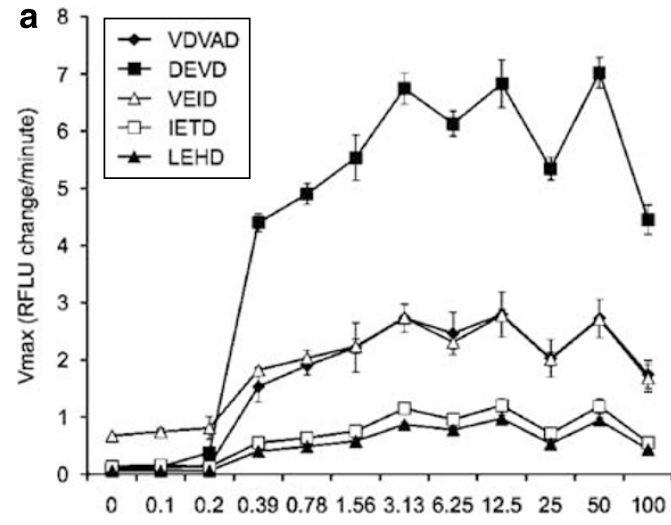

[Cytochrome $c](\mu \mathrm{M})$

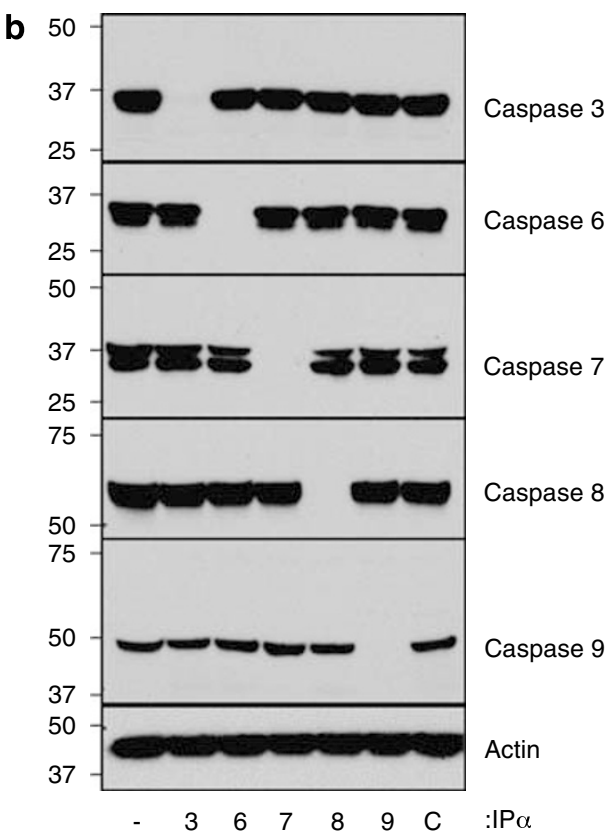

Figure 1 Caspase activity in cytosolic extracts depleted of caspases prior to incubation with cytochrome $c$ and dATP. (a) Jurkat cytosolic extract $(100 \mu \mathrm{g})$ was incubated with the indicated concentrations of cytochrome $c$ and dATP $(1 \mathrm{mM})$ for $30 \mathrm{~min}$ at $37^{\circ} \mathrm{C}$. Treated extracts were then assessed for all caspase activities for $30 \mathrm{~min}$ at $37^{\circ} \mathrm{C}$, reading every minute. All substrates are AFC conjugated and only the peptide sequence is shown. Values represent mean \pm S.D. of quadruplicate readings from one experiment and one representative of three independent experiments. (b) Immunoblots of immunodepleted Jurkat cytosolic extracts. Immunodepleted caspases $(\mathrm{IP} \alpha)$ are represented by the corresponding number. Extracts without immunodepletion are represented by '-'. C refers to extract immunodepleted with either mouse or rabbit immunoglobulin $G$. The blot is representative of three independent experiments

cytochrome $c$ were dependent on caspase-3 itself, or caspases activated downstream of it. Small decreases in caspase activity in caspase-7-depleted extracts, for VDVAD and DEVD, were observed, accounting for the incomplete loss of cleavage activity in caspase-3-depleted extracts. Caspase2 (Supplementary Figure 2b), -6 or -8 immunodepletion (Table 2), prior to addition of cytochrome $c$ and dATP did not remove significant amounts of any induced substrate cleavage. 
Table 2 Caspase activity in extracts immunodepleted of caspases prior to incubation with cytochrome $c$ and dATP

\begin{tabular}{|c|c|c|c|c|c|}
\hline \multirow[b]{3}{*}{ Immunodepleted extract $^{a}$} & \multicolumn{5}{|c|}{ Substrate (AFC) } \\
\hline & VDVAD & DEVD & VEID & IETD & LEHD \\
\hline & \multicolumn{5}{|c|}{ Substrate cleavage (mean RFLU change/min $/ \mu \mathrm{g} \pm \mathrm{S}^{\mathrm{D}} \mathrm{D}^{\mathrm{b}}$ ). } \\
\hline Control $^{\mathrm{C}}$ & 0.00 & 0.00 & 0.04 & 0.00 & 0.00 \\
\hline No antibody & 0.11 & $0.28 \pm 0.01$ & 0.10 & 0.02 & 0.02 \\
\hline Caspase-3 & 0.02 & 0.06 & 0.03 & 0.00 & 0.00 \\
\hline Caspase-6 & 0.10 & 0.26 & 0.08 & 0.02 & 0.02 \\
\hline Caspase-7 & $0.08 \pm 0.01$ & $0.21 \pm 0.01$ & 0.09 & 0.02 & 0.02 \\
\hline Caspase-8 & 0.11 & $0.28 \pm 0.01$ & 0.10 & 0.02 & 0.02 \\
\hline Caspase-9 & 0.01 & 0.02 & 0.03 & 0.00 & 0.00 \\
\hline Mouse IgG & 0.10 & $0.27 \pm 0.01$ & $0.10 \pm 0.01$ & 0.02 & 0.02 \\
\hline Rabbit IgG & 0.12 & $0.31 \pm 0.01$ & $0.10 \pm 0.01$ & 0.02 & 0.02 \\
\hline
\end{tabular}

${ }^{a}$ Extracts were depleted with antibody to the indicated caspase (or control IgG) and incubated with $100 \mu \mathrm{M}$ cytochrome $c / 1 \mathrm{mM} \mathrm{dATP}, 30 \mathrm{~min}, 37^{\circ} \mathrm{C}$. ${ }^{\mathrm{b}} \mathrm{Only}$ standard deviations that were significant to two decimal places are shown. ${ }^{\mathrm{C}}$ Extract without antibody, incubated without cytochrome $c$ or dATP

Caspases directly cleave substrates, or can act indirectly by activating other caspases, for example, caspase-3 cleavage of caspase- $6 .{ }^{18}$ Therefore, caspase immunodepletions were performed after cytochrome $c$ incubation with cytosolic extract. Western blot analysis of the immunodepleted extracts demonstrated that all full-length and processed forms of caspases were absent (Figure $2 \mathrm{a}$ and Supplementary Figure 3a). Immunodepletion of caspase-3 after activation removed VDVAD (Figure 2bi) and DEVD (Figure 2bii) cleavage. Immunodepletion of active caspase- 6 removed VEID-cleaving activity to the same extent as caspase- 3 immunodepletion (Figure 2biii) and removed more IETD and LEHD cleavage activity than did removal of caspase-3 (Figure 2 biv and $v$ ). The removal of these substrate cleavage activities from caspase6-depleted cytosolic extracts corresponds to the cleavage activities that recombinant caspase-6 displayed (Table 1). Immunodepletion of caspase-7 partially removed the VDVAD and DEVD cleavage activity (Figure 2bi and ii), and was not comparable to cytosolic extracts depleted of active caspase-3. Immunodepletion of caspase-2, -8 or -9 did not deplete VDVAD (Supplementary Figure 3bi), IETD or LEHD (Figure 2biv and v) cleavage activity respectively. In all cases, the caspase-cleaving activities of the immunoprecipitates corresponded to the activities that were absent from the immunodepleted cytosolic extracts. All tested substratecleavage activities were associated with caspase-3 immunoprecipitations (Figure 2b), VDVAD (Figure 2bi) and DEVD (Figure 2bii) cleavage with caspase-7 immunodepletion and VEID, IETD and LEHD substrates with caspase-6 immunoprecipitation (Figure 2biii-v). These experiments demonstrated that caspase-3 is the most critical caspase in terms of cleavage of synthetic reporter substrates either because of its own activity, in the case of VDVAD, DEVD and to some extent VEID, or on its ability to cleave and activate caspase$6,^{18}$ which cleaves VEID, IETD and LEHD. Surprisingly, caspase- 6 seems to cleave more substrates than caspase- 7 in this system.

The same model of cytochrome $c$ activation of the apoptosome and caspase activation in cytosolic extract was used to test a panel of caspase-specific inhibitors. Incubation of inhibitors with cytosolic extracts prior to addition of cytochrome $c$ and dATP was followed by determination of substrate-cleavage activity (Figure $3 a$ ). Cytochrome $c$ induc- tion of all substrate cleavage was completely inhibited by the pan-caspase inhibitors zVAD-FMK and qVD-OPH. Using two doses of the caspase-specific inhibitors revealed a general pattern of inhibition. All inhibitors completely abrogated all substrate cleavage at $25 \mu \mathrm{M}$. However, at $5 \mu \mathrm{M}$ inhibitor differences between inhibitors were revealed. Specifically, zVDVAD-FMK did not inhibit any substrate-cleavage activity more than $25 \%$ of total activity. Also, zVEID-FMK and zLEHDFMK were poor inhibitors at this dose, achieving 50-75\% inhibition of all substrates. The most efficient caspase-specific inhibitors were zDEVD-FMK and zIETD-FMK that were able to inhibit almost completely at the $5 \mu \mathrm{M}$ dose, similar to the pan-caspase inhibitors.

Inhibition of upstream caspases will naturally inhibit activation of downstream caspases, and therefore we compared the effects of adding inhibitors after cytochrome $c$ had induced caspase activity in this system. The resulting set of inhibitory profiles (Figure $3 b$ ) obtained with all substrates was similar to that obtained when inhibitors were added prior to cytochrome $c$ and dATP. All inhibitors were able to completely abrogate all substrate cleavage at $25 \mu \mathrm{M}$. At $5 \mu \mathrm{M}$, zVDVAD-FMK was the least effective inhibitor, with zVEID-FMK and zLEHD-FMK only able to partially inhibit all substrate cleavage. The pancaspase and ZDEVD-FMK and ZIETD-FMK inhibitors were able to almost completely abolish all substrate cleavage. This indicates that the inhibitors did not prevent substrate cleavage only by inhibiting caspase- 9 activity. As the inhibitors have the same efficiency before or after cytochrome $c$ addition, it is likely that the inhibitors inactivated the same targets in each case. These results confirm that putative-specific caspase inhibitors can target caspases other than those that they are thought to specifically inhibit. The effects of these putative caspase-specific inhibitors on caspase- 3 and -6 could result in inhibition of all caspase activity as monitored by substrate cleavage.

Inhibition of apoptosis by putative caspase-specific inhibitors. Using treatments that induce both intrinsic and extrinsic pathways of apoptosis, the selectivity of each specific caspase inhibitor was then determined in cells. To engage the extrinsic pathway an agonistic anti-Fas antibody acts through activation of caspase- 8 by the DISC and activates the mitochondrial pathway of apoptosis through 


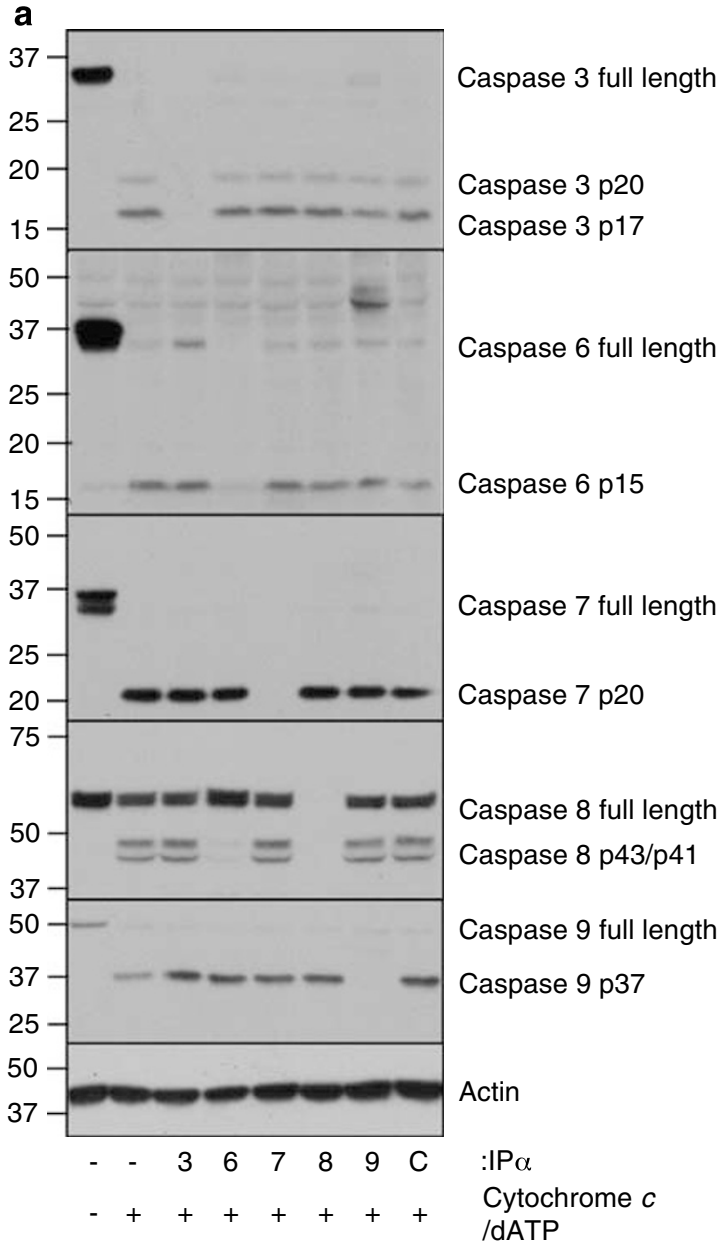

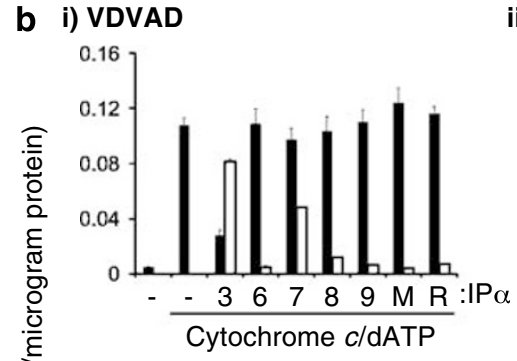
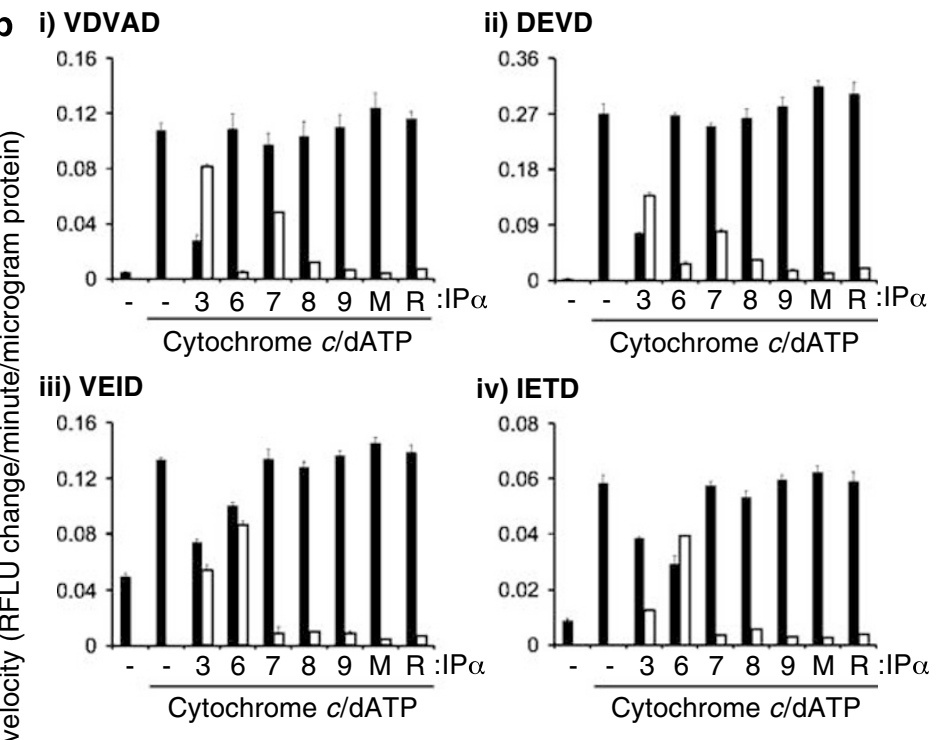

v) LEHD

है
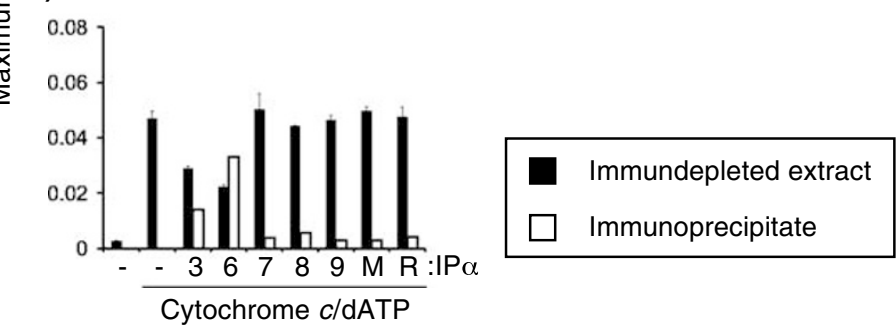

Figure 2 Caspase activity in Jurkat cytosolic extracts incubated with cytochrome $c$ and dATP followed by immunodepletion. (a) Caspase immunoblots of Jurkat cytosolic extract following cytochrome $c$ and dATP incubation. Immunodepleted caspases $(\mathrm{IP} \alpha)$ are represented by the corresponding number. Extracts without immunodepletion are represented by '-'. C refers to extract immunodepleted with either mouse or rabbit immunoglobulin G. (b) Caspase activities of immunodepleted Jurkat cytosolic extracts (black bars) and the corresponding immunoprecipitates (white bars) were measured using fluorogenic substrates and the rate of initial cleavage was plotted as the caspase cleaving activity. The numbers represent immunoprecipitated caspase (IP $\alpha)$ as in (a). Immunoprecipitations using mouse or rabbit immunoglobulin $\mathrm{G}$ are represented by $\mathrm{M}$ or $\mathrm{R}$ respectively. All substrates are AFC conjugated and only the peptide sequence is shown. The substrate cleaving activity of the immunoprecipitated caspase was determined by incubating $5 \%$ of the protein $A / G$ resin (a volume equivalent to an equal amount of cytosolic extract) with each fluorogenic substrate. Values in the immunodepleted extracts represent the mean + S.D. of four replicates of one experiment, representative of two independent experiments and values in the immunoprecipitates represent the mean \pm S.D. of two replicates of one experiment, representative of two independent experiments

cleavage of BID and cytochrome $c$ release ${ }^{6,7}$ After overnight treatment with the anti-Fas antibody approximately $80 \%$ apoptosis was observed (Figure 4a). In cells preincubated with pan-caspase or caspase-specific inhibitors prior to antiFas antibody, varying amounts of apoptosis were observed. The pan-caspase inhibitors ZVAD-FMK and qVD-OPH inhibited apoptosis to the greatest extent. The most potent 'specific' inhibitor was zIETD-FMK, most likely through inhibition of caspase-8 as well as caspases-3 and -6 . The inhibitors zDEVD-FMK, zVEID-FMK and ZLEHD-FMK all inhibited apoptosis partially and this may be due to incomplete inhibition of caspases in the cascade. The poorest inhibitor was ZVDVAD-FMK, either due to an inability to inhibit any caspases in the pathway or poor membrane permeability. To discount the potential instability of these inhibitors over long periods of time and potentially poor membrane permeability, a shorter incubation time after
anti-Fas antibody treatment was also assessed for annexin $\mathrm{V}$ staining after $1 \mathrm{~h}$ preincubation with the inhibitors. After $6 \mathrm{~h}$ treatment, approximately $50 \%$ apoptosis was observed and was blocked by all of the inhibitors tested, except ZVDVADFMK (Figure 4c). Although all of these caspase inhibitors may inhibit their 'specific' caspase, it cannot be presumed that they act in this way, considering the other data presented.

Intrinsic apoptosis induced by UV irradiation is a wellcharacterized pathway depending on mitochondrial release of cytochrome $c$, resulting in activation of caspase-9, then caspases- 3 and $-7 .{ }^{20}$ The pan-caspase inhibitors were the most efficient inhibitors of apoptosis (Figure $4 \mathrm{~b}$ ). The best caspase-specific inhibitors were zDEVD-FMK, zIETD-FMK and zVEID-FMK. However, zVDVAD-FMK and more surprisingly zLEHD-FMK, the putative caspase-9 specific inhibitor, were virtually ineffective at inhibiting apoptosis. Using shorter 


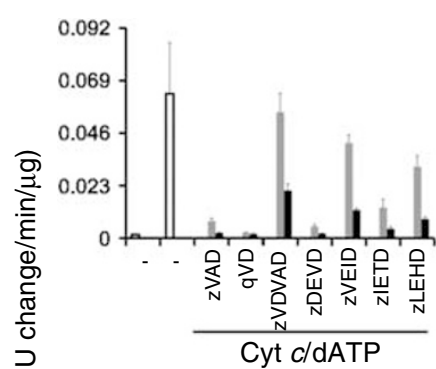

iii) VEID

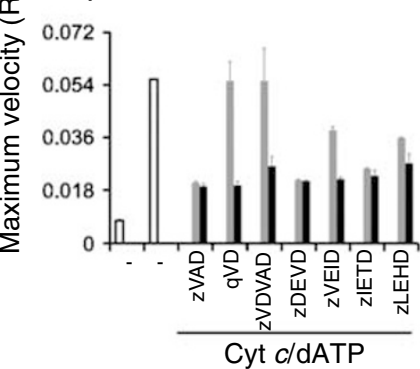

v) LEHD

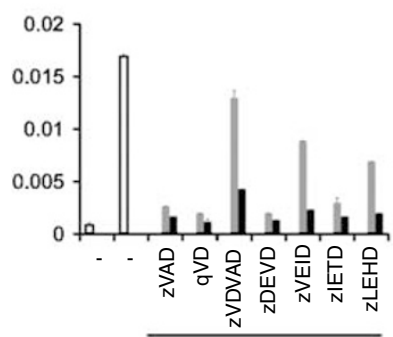

Cyt $c /$ dATP ii) DEVD

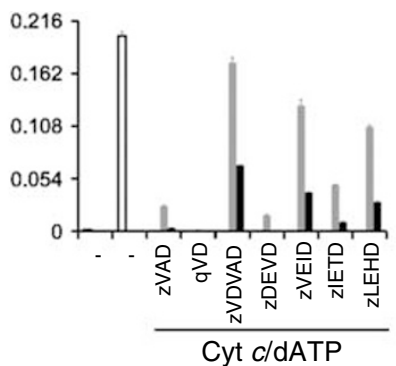

iv) IETD

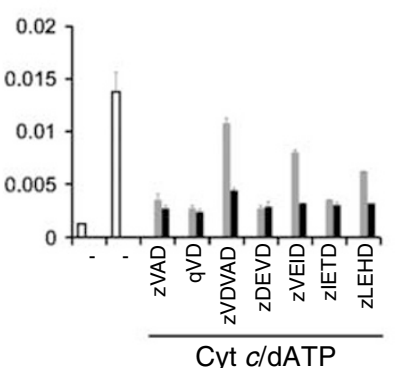

Cyt $c / d A T P$ b i) VDVAD

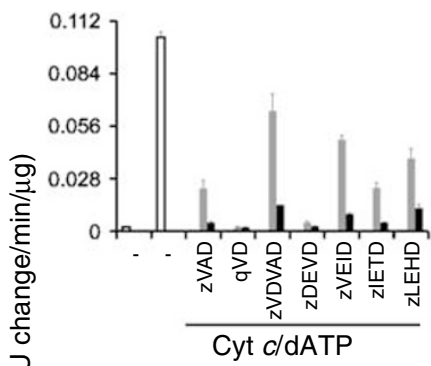

iii) VEID

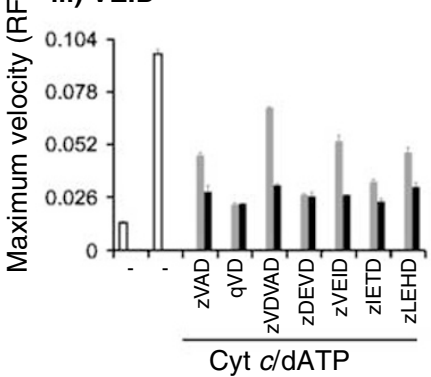

v) LEHD

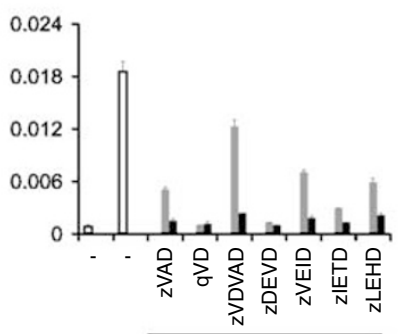

Cyt $c /$ dATP ii) DEVD

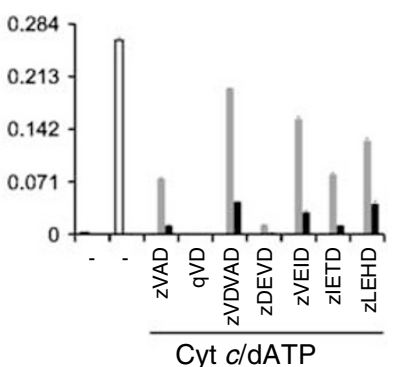

iv) IETD

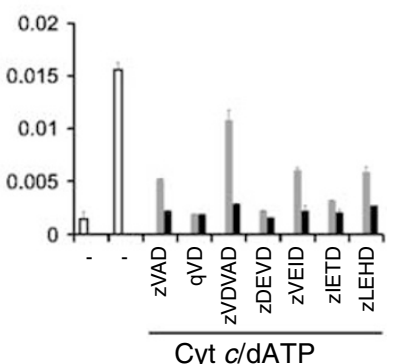

Cyt c/dATP

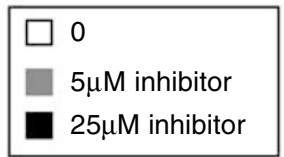

Figure 3 Inhibition of cytochrome $c$ and dATP-induced caspase activity with caspase-specific inhibitors. (a) Cytosolic extracts from Jurkat cells were preincubated with caspase inhibitor as indicated for $15 \mathrm{~min}$ prior to the addition of cytochrome $c$ and dATP to initiate caspase activity for $30 \mathrm{~min}$ at $37^{\circ} \mathrm{C}$. (b) Cytosolic extracts from Jurkat cells were incubated with cytochrome $c$ and dATP to initiate caspase activity for $30 \mathrm{~min}$ at $37^{\circ} \mathrm{C}$. Inhibitors were added to the incubated samples at 5 (gray bars) or $25 \mu \mathrm{M}$ (black bars) and incubated for $45 \mathrm{~min}$ more at $37^{\circ} \mathrm{C}$. All inhibitors are $\mathrm{O}$-methylated and FMK conjugated and only the peptide sequence is shown. Fluorogenic substrates were added to the extracts and generation of fluorophore was followed for $30 \mathrm{~min}$, every minute, at $37^{\circ} \mathrm{C}$. All substrates are AFC conjugated and only the peptide sequence is shown. The initial rate was taken as measurement of caspase activity. Values represent the mean \pm S.D. of four replicates and are representative of two independent experiments

time points after UV treatment ( $6 \mathrm{~h}$ ) and longer preincubation with caspase inhibitors $(1 \mathrm{~h})$ cells were assessed for annexin $\mathrm{V}$ staining. As with the longer time point, only qVD-OPH completely inhibited apoptosis induced by UV (Figure 4c). Other inhibitors were poor blockers of apoptosis induced by this stimulus.

These experiments demonstrated that FMK-based caspase inhibitors are unreliable tools for monitoring specific caspase in cells. The putative caspase-8 specific inhibitor, zIETD-FMK, was able to inhibit anti-Fas-induced apoptosis as expected, but not as efficiently as the pan-caspase inhibitors. On the other hand, the putative caspase-9-specific inhibitor, zLEHD-FMK, was virtually ineffective at inhibiting UV-induced apoptosis, a pathway prominently dependent on caspase- $9 .^{20}$ Another reason for the lack of effects associated with FMKbased inhibitors may be due to poor uptake into cells. However, all inhibitors, except zVDVAD were able to inhibit apoptosis induced by the anti-Fas antibody indicating these are fully permeable to the cytosol of the cell. It is difficult to determine the permeability of zVDVAD-FMK, as it is only able to partially inhibit apoptosis in cells and caspase activity in a cell-free system (Figures 3 and 4).

Apoptosis in caspase-deficient cells. To further examine the specificity of caspase substrates and inhibitors Jurkat cells lacking procaspase- 9 (JMR) and a control cell line with caspase-9 restored (F9) were utilized. ${ }^{21}$ Induction of apoptosis using the agonistic anti-Fas antibody induced VDVAD, DEVD and LEHD cleavage in cytosolic extracts from cells with or without caspase-9 protein (Figure 5a). In cells lacking caspase-9, an increase in LEHD cleavage activity may have been unexpected. However, recombinant human caspase-3, -6 and -8 can cleave LEHD better than caspase- 9 itself (Table 1), and activation of these caspases is induced in this scenario. ${ }^{8}$ The ability of zLEHD-FMK to inhibit apoptosis in caspase- 9 deficient cells was also determined. Treatment of caspase-9-deficient cells or those with restored caspase- 9 with anti-Fas antibody-induced apoptosis in both cases (30 versus 45\% respectively; Figure 5b). zLEHD-FMK inhibited apoptosis in a dose- 

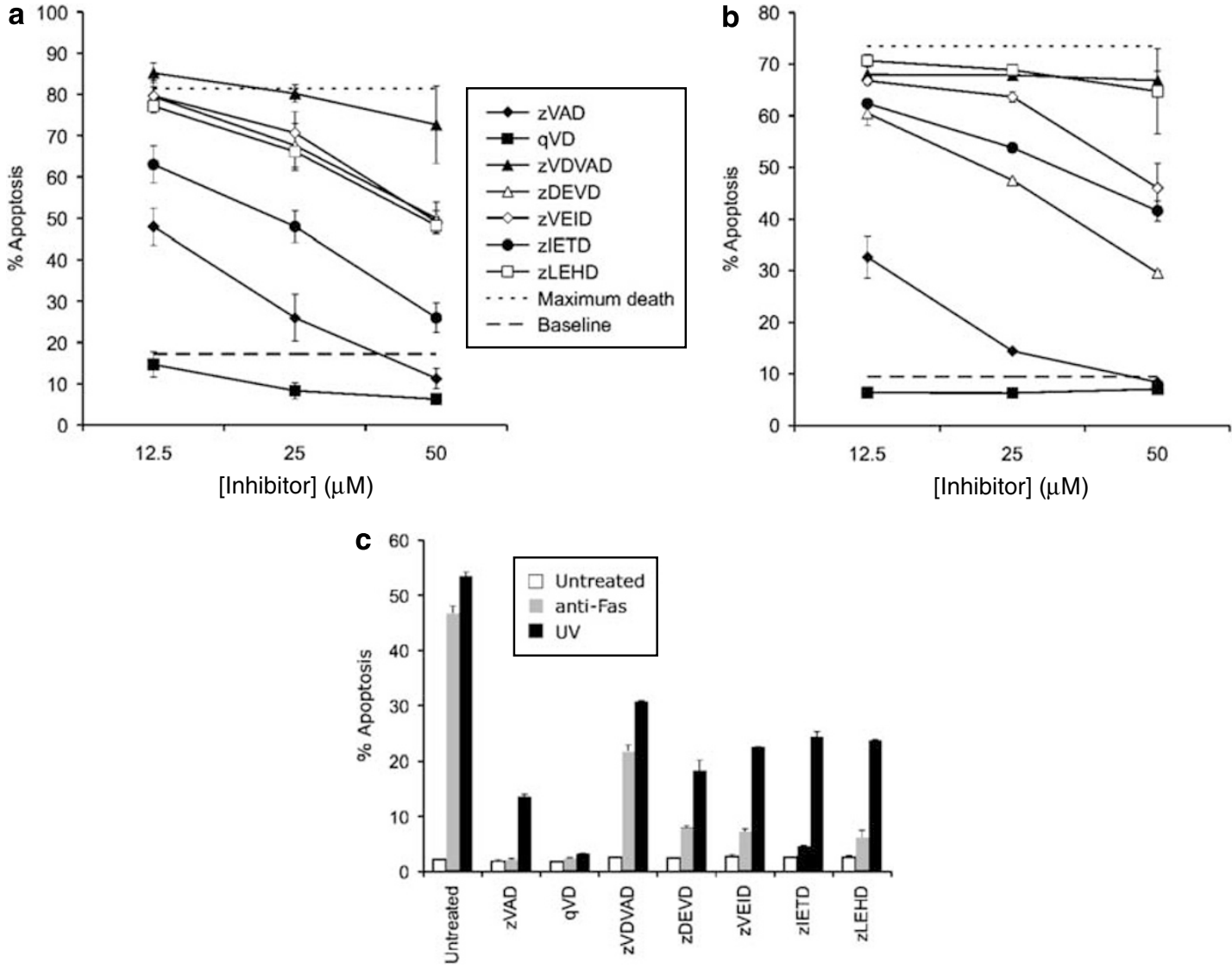

Figure 4 Inhibition of apoptosis by putative caspase-specific inhibitors. Jurkat cells $(500000 / \mathrm{ml})$ were incubated with the indicated concentrations of caspase inhibitor for $15(\mathbf{a}, \mathbf{b})$ or 60 (c) minutes at $37^{\circ} \mathrm{C}$. All inhibitors are $\mathrm{O}$-methylated and FMK conjugated and only the peptide sequence is shown. Apoptosis was induced by treating cells with $30 \mathrm{ng} / \mathrm{ml}$ agonistic anti-Fas antibody (a) or $35 \mathrm{~mJ}$ ultraviolet irradiation (b) and incubated for $6 \mathrm{~h}$ at $37^{\circ} \mathrm{C}$. Alternatively, cells were treated with $50 \mathrm{ng} / \mathrm{ml}$ agonistic anti-Fas antibody or $35 \mathrm{~mJ}$ ultraviolet irradiation and incubated overnight for $18 \mathrm{~h}$ at $37^{\circ} \mathrm{C}$ (c). Apoptosis was assessed using annexin V-FITC. Values represent the mean \pm S.D. from two replicates, representative of two independent experiments

dependent manner in both cell lines. At the lowest dose tested, zLEHD-FMK was fully able to inhibit apoptosis in the caspase-9-deficient cells. In contrast, in caspase-9expressing cells apoptosis could only be fully inhibited at $100 \mu \mathrm{M}$ most likely due to the greater efficiency of caspase activation in the presence of caspase-9. Absence and presence of functional caspase- 9 was confirmed by Western blotting of JMR and F9 cells after treatment with anti-Fas (Figure 5c). These results once again point to the substrates as not being exclusive for one specific caspase, especially in a cellular context.

\section{Discussion}

The use of peptide sequences derived from substrates of proteases as inhibitors or means to monitor activity is a classic technique to determine the involvement of proteases in specific pathways. These peptide-based substrates and inhibitors were quickly adopted as the standard reagents to determine the involvement of caspases in apoptotic pathways. These substrates and inhibitors are now readily available from many commercial sources and are marketed as caspase-specific reagents. By comparing many caspase cleavage sites the diversity of a cleavage site for a specific caspase can be appreciated and the lack of selectivity that commercially available substrates and inhibitors possess must be noted.

Defining the roles of caspases has been a prevalent feature of apoptosis research since the components of the apoptotic pathways were elucidated. Sophisticated approaches have been employed to achieve this goal including the use of caspase-deficient cells from knockout mice, variants of cell lines lacking caspases or the use of RNA interference. Unfortunately these approaches are not without problems, as genetic knockouts of caspases in mice have been shown to alter the expression profiles of other caspases resulting in complimentary activity ${ }^{22}$ and also display strain-dependent phenotypes. $^{23}$ The use of RNA interference to reduce caspase expression also has benefits and drawbacks. On one hand, reduction of caspase levels early after knockdown should provide an ideal environment for testing the absence of the caspase, as there will be less chance for a cell to compensate for the absent caspase. However, problems with RNA interference technology relate to alternative signaling induced by short RNA species and off-target effects. Proper controls must be utilized in these situations to ensure that caspase expression has not been affected. ${ }^{24}$ Our laboratory has previously used an in vivo labeling technique to determine 

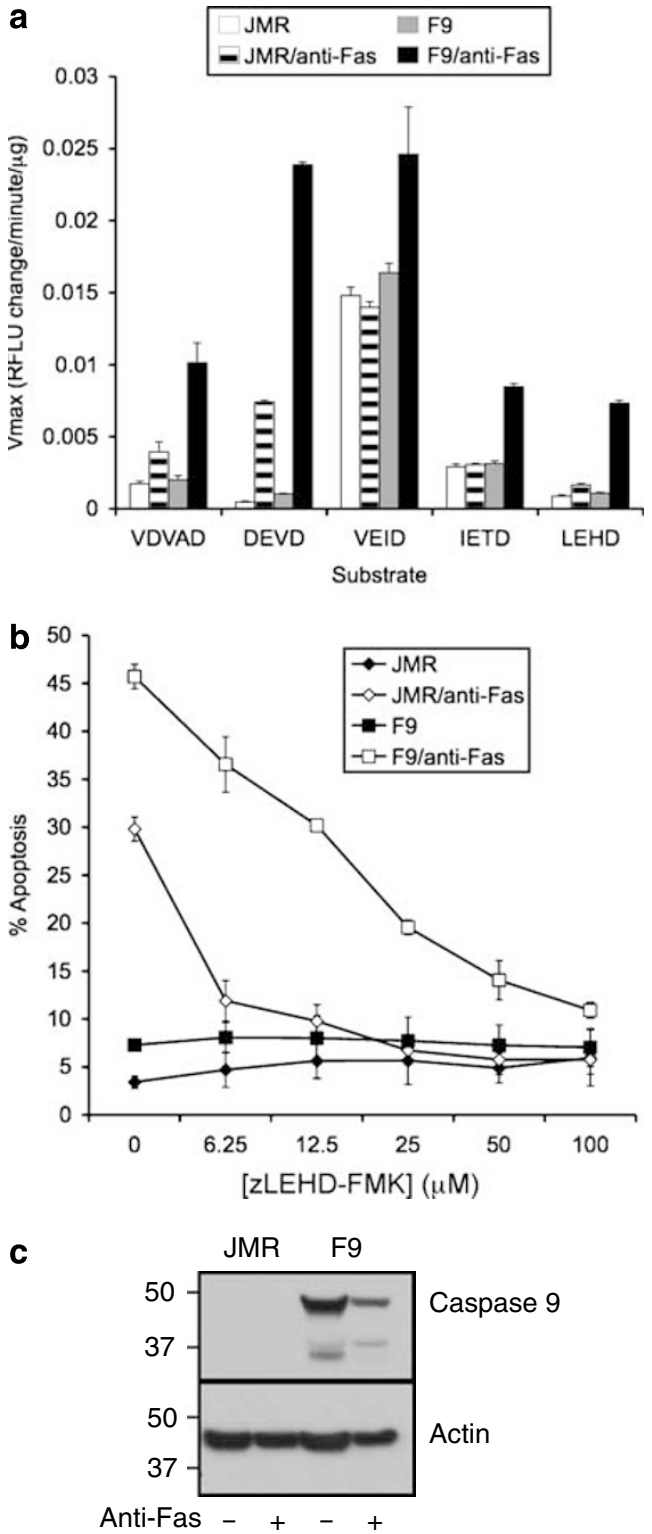

Figure 5 Caspase activities in caspase deficient cells. (a) Caspase-9-deficient Jurkat cells (JMR) or caspase-9-deficient Jurkat cells with caspase-9 restored (F9) were treated with $100 \mathrm{ng} / \mathrm{ml}$ agonistic anti-Fas antibody for $18 \mathrm{~h}$ at $37^{\circ} \mathrm{C}$. Cells were harvested and extracts prepared by freeze-thaw. Caspase activity was assessed by adding fluorogenic substrates to the extracts and monitoring generation of the fluorophore every minute for $30 \mathrm{~min}$ at $37^{\circ} \mathrm{C}$. All substrates are AFC conjugated and only the peptide sequence is shown. The initial rate per $\mu \mathrm{g}$ protein was plotted as a measure of activity. Values represent the mean \pm S.D. of four replicate readings, representative of two independent experiments. (b) JMR or F9 cells were preincubated with zLEHD-FMK for $15 \mathrm{~min}$ at $37^{\circ} \mathrm{C}$. Apoptosis was induced by the addition of $30 \mathrm{ng} / \mathrm{ml}$ agonistic anti-Fas antibody for $18 \mathrm{~h}$ at $37^{\circ} \mathrm{C}$. Apoptosis was assessed by annexin V-FITC staining. Values represent the mean \pm S.D. from two replicate reading, representative of two independent experiments. (c) Western blot of JMR (caspase-9 deficient) and F9 (caspase-9 reconstituted) cells after treatment with $500 \mathrm{ng} / \mathrm{ml}$ anti-Fas. The immunoblot is representative of two independent experiments

the first caspase activated in an apoptotic setting in a nondiscriminatory manner. ${ }^{20}$ Biotinylated VAD-FMK-bound caspases activated by different apoptotic stimuli indicating that full-length caspase-2, -8 and -9 were able to initiate apoptosis induced by heat shock, anti-Fas and UV irradiation respectively. However, biotinylated VAD-FMK is very inefficient at binding to full-length caspases and inhibiting downstream caspase cleavage. ${ }^{25}$ More specific inhibitors of individual caspases have been designed to overcome the problems of current caspase-specific inhibitors, but they still possess some overlap in their specificities, ${ }^{12}$ especially taking into account the relative amounts of caspase- 3 in a cell after induction of apoptosis. ${ }^{26,27}$

Our conclusions have been based on analysis of recombinant caspases, as well as native caspases activated in vitro and in cells. The recombinant initiator caspases used in this study were truncated to simplify purification and increase the activity of the proteins. While active site titration was used to ensure that equivalent activities of these truncated caspases were compared, it is formally possible that they do not represent the precise substrate specificities or relatively activities of those found in cells. We found that the executioner caspases-3, -6 and -7 , have the same substrate specificity either as recombinant proteins or isolated from cytosolic extracts. However, we were unable to demonstrate activity of the initiator caspases from cytosolic extracts in terms of substrate cleavage. As initiator caspases have the pro-domain removed through cleavage, we suggest that the truncated initiator caspases that we used in our study represent a form of active caspase found in apoptotic cells. Removal of the prodomain by caspase cleavage seems to be responsible for stabilizing activity of the caspase when full-length caspases are compared to $\mathrm{N}$-terminally truncated versions, ${ }^{28}$ rather than altering its specificity, although the latter has not been tested formally. While some reports claim that the full-length and processed forms of caspase- 8 show a difference in substrate specificity, ${ }^{29,30}$ these studies attribute the former to nonapoptotic functions of caspase-8. They suggest that only processed, and not full-length caspase-8 is able to cleave caspase-3 as an apoptotic initiation step. In any case, we suggest that such considerations do not profoundly impact on our conclusions regarding the use of 'specific' substrates or inhibitors for the identification of caspase activity.

Using specific immunodepletion of caspases circumvents the problems of genetic manipulation of caspase expression and can be used confidently to discern roles of each caspase in an in vitro setting. From these experiments the prevalence of caspase-3 activity induced by cytochrome $c$ activation of the apoptosome was striking, compared to the requirements of caspase- 6 or -7 (Table 2), consistent with the activities that these recombinant enzymes possess alone. This also confirms that caspase- 6 is activated by caspase- 3 and not by caspase- 9 or -7 directly, consistent with other reports. ${ }^{18}$ There was no effect after immunodepletion of caspase-2 (Supplementary Figures 2 and 3) or -8 (Table 2 and Figure 2) from extracts, suggesting that there is either little or no activity associated with these caspases after activation of the apoptosome with cytochrome $c$. It can also be speculated that there is no involvement of caspase-10 activity in this caspase cascade as virtually all activity can be accounted for by removal of caspase- $3,-6$ or -7 . This immunoprecipitation procedure of active caspases may therefore be a useful tool to determine which caspases are active in a given scenario of apoptosis. 
In summary, our report demonstrates that caspase-specific substrates and inhibitors lack the proposed specificity that is associated with these reagents. Therefore, caution is needed when using these reagents alone. Our experiments demonstrate that caspase-3 is the most promiscuous and efficient caspase among the executioner caspases and its activity is the most prevalent after engagement of the mitochondrial pathway of apoptosis. It is because of these properties of caspase-3 that putative caspase-specific substrates and inhibitors are unreliable tools for monitoring an individual caspase's contribution to an apoptotic pathway.

\section{Materials and Methods \\ Materials. Full-length recombinant caspase-3, -6 and -7 were C-terminally tagged with a hexahistidine motif and were isolated according to standard protocols. ${ }^{31,32}$ Truncated caspase-2 (lacking the caspase recruitment domain (CARD)), caspase-8 (lacking the two death-effector domains (DED)), caspase-9 (lacking the CARD) and caspase-10a (lacking the DEDs) C-terminally tagged with a hexahistidine motif were isolated using the same protocols. Specific activity of the caspases was quantified by titrating ZVAD-FMK (non-O-methylated) with recombinant caspase and monitoring activity against an 7-amido-4- (trimethyl)coumarin (AFC)-conjugated substrate, caspase-2 - VDVAD, caspase-3 - DEVD, caspase-6 - VEID, caspase-7 - DEVD, caspase-8 - IETD, caspase-9 - LEHD and caspase-10a - IETD. ${ }^{31,32}$ Antibodies used for Western blotting were caspase-2 (1:1000 dilution from Chemicon, Temecula, CA, USA), caspase-3 (1:200 dilution from BD, Franklin Lakes, NJ, USA), caspase-6 (1:1000 dilution from Cell Signaling Technology, Danvers, MA, USA), caspase-7 (1:1000 dilution from Cell Signaling Technology), caspase-8 (1:100 dilution kindly provided by $\mathrm{Dr}$ Marcus Peter, University of Chicago, IL, USA), caspase-9 (1: 1000 dilution from Cell Signaling Technology) and caspase-10 (1:1000 dilution from MBL International, Woburn, MA, USA). Antibodies for immunodepletion were caspase-2 (Santa Cruz Biotechnology, Santa Cruz, CA, USA, clone H-19), caspase-3 (BD clone 19), caspase-6 (Upstate, Charlottesville, VA, USA), caspase-7 (BD clone 51), caspase-8 (Axxora, San Diego, CA, USA), caspase-9 (Cayman Chemicals, Ann Arbor, Ml, USA). N-terminally acetylated fluorogenic caspase substrates were prepared as a $20 \mathrm{mM}$ stock in DMSO. VDVAD-AFC, DEVD-AFC, VEID-AFC, IETD-AFC and LEHD-AFC were purchased from Calbiochem (San Diego, CA, USA). Pan- or specific fluoromethylketone (FMK)-conjugated caspase inhibitors were prepared as a $10 \mathrm{mM}$ stock in DMSO. zVAD-FMK, Z-VAD-FMK (non-O-methylated) and qVD- $\mathrm{OPH}$ were purchased from MP Biomedicals (Solon, OH, USA). Caspase-specific inhibitors zVDVAD-FMK, zDEVD-FMK, zVEID-FMK, zIETD-FMK, zLEHD-FMK (all O-methylated) were purchased from Calbiochem.}

Cell lines, culture, apoptosis induction and cytosol preparation. Jurkat cells were cultured in RPMI-1640 supplemented with $10 \%$ FBS, 50 I.U. Penicillin, $50 \mu \mathrm{g} / \mathrm{ml}$ Streptomycin, $2 \mathrm{mM}$ Glutamine, $1 \mathrm{mM}$ 4-(2hydroxyethyl)piperazine-1-ethanesulfonic acid (HEPES). Apoptosis was induced by agonistic anti-Fas antibody $\mathrm{CH}-11$ (Upstate) or ultraviolet irradiation. Cells were incubated and harvested at various time points, usually 6 or $18 \mathrm{~h}$. Cytosolic preparations were prepared by washing cells with PBS, then swelling buffer $(10 \mathrm{mM}$ HEPES, pH 7.0, $5 \mathrm{mM} \mathrm{MgCl}_{2}, 0.67 \mathrm{mM}$ dithiothreitol (DTT), complete protease inhibitors (Roche Applied Science, Indianapolis, IN, USA)). Cells were swollen in an equal volume of swelling buffer for $15 \mathrm{~min}$ on ice. Cells were disrupted by passing through a 22-gauge needle 10 times, until $>80 \%$ of nuclei-stained positive with trypan blue. The suspension was centrifuged twice at $15000 \times g$ for $30 \mathrm{~min}, 4^{\circ} \mathrm{C}$. The supernatant was centrifuged at $100000 \times g, 1 \mathrm{~h}, 4^{\circ} \mathrm{C}$ to obtain the $S 100$ fraction. The $\mathrm{S} 100$ fraction was filtered by centrifugation at $4^{\circ} \mathrm{C}$. Protein concentration was determined by absorbance at $280 \mathrm{~nm}$. Extracts from apoptotic cells were prepared by freeze-thawing three times in a dry ice - methanol bath. The extracts were centrifuged at $15000 \times \mathrm{g}, 4^{\circ} \mathrm{C}, 15 \mathrm{~min}$ and the protein concentration was determined by absorbance at $280 \mathrm{~nm}$.

Recombinant caspase activity. Purified recombinant caspase $(10 \mu \mathrm{l}$ of $200 \mathrm{nM}$ ) was incubated with $100 \mu \mathrm{M}$ substrate at $37^{\circ} \mathrm{C}$ for $30 \mathrm{~min}$ using a Spectrafluor Fluorescence Plate Reader with excitation at $400 \mathrm{~nm}$ and emission at $505 \mathrm{~nm}$. The initial linear rate of the generation of the fluorophore was used to determine the maximum velocity of the reaction and was used as a measure of caspase activity against the substrate.

In vitro activation of the apoptosome by cytochrome $c$. Jurkat $S 100$ $(100 \mu \mathrm{g}$ in $10 \mu \mathrm{l})$ was incubated with horse heart cytochrome $c(1 \mathrm{mM}$ stock in water; Sigma, St. Louis, MO, USA) and $1 \mathrm{mM}$ dATP (10 mM stock in water; Sigma) for $30 \mathrm{~min}$ at $37^{\circ} \mathrm{C}$. Caspase-induced activity was measured using $10 \mu \mathrm{l}$ of the activated sample with $90 \mu \mathrm{l}$ of $100 \mu \mathrm{M}$ caspase substrate and measured the same as recombinant caspases.

Immunodepletion of caspases from Jurkat S100. Antibodies were incubated with protein-A/G agarose $(40 \mu \mathrm{l}$ of a 50:50 resin; Santa Cruz Biotechnology) for $3 \mathrm{~h}$ at $4^{\circ} \mathrm{C}$. Cytosolic $\mathrm{S} 100$ fraction $(1 \mathrm{mg}$ in $100 \mu \mathrm{l})$ was rotated with the antibody bound beads at $4{ }^{\circ} \mathrm{C}$, overnight. The resin was separated from the $\mathrm{S} 100$ fraction by centrifugation $\left(1 \mathrm{~min}, 4^{\circ} \mathrm{C}, 1000 \times \mathrm{g}\right)$. The supernatant was filtered by centrifugation to ensure complete removal of the resin. The supernatant was used for in vitro activation assays as above or was directly measured for caspase activity if already incubated with cytochrome $c$ and dATP. Immunoprecipitates were washed once with swelling buffer and the resin was directly added to caspase buffer and activity was measured as described above.

Analysis of apoptotic cells. Treated cells were harvested and directly resuspended into annexin $\mathrm{V}$ binding buffer $(150 \mathrm{mM} \mathrm{NaCl}, 10 \mathrm{mM}$ HEPES, $\mathrm{pH} 7.4$, $5 \mathrm{mM} \mathrm{KCl}, 1.8 \mathrm{mM} \mathrm{CaCl}_{2}, 1 \mathrm{mM} \mathrm{MgCl}_{2}, 1 \%$ Annexin V-fluorescein isothiocyanate (FITC) conjugate (Invitrogen, Carlsbad, CA, USA)) and incubated for 10 min at $4^{\circ} \mathrm{C}$. Annexin V positive cells were determined using the FL-1 channel of a FACScan II (BD) on high setting. Extracts from freeze-thawed apoptotic cells were incubated with caspase assay buffer containing different substrates and activity was measured as described above.

Acknowledgements. We thank Dr. Ingo Schmitz (University of Düsseldorf) for providing the caspase-9-deficient, JMR, and caspase-9 reconstituted, F9, Jurkat cells. This work was financially supported by Grants Al47891 and CA69381 from the United States National Institute of Health.

1. Boatright KM, Renatus M, Scott FL, Sperandio S, Shin H, Pedersen IM et al. A unified model for apical caspase activation. Mol Cell 2003; 11: 529-541.

2. Timmer JC, Salvesen GS. Caspase substrates. Cell Death Differ 2007; 14: 66-72.

3. Li P, Nijhawan D, Budihardjo I, Srinivasula SM, Ahmad M, Alnemri ES et al. Cytochrome c and DATP-dependent formation of Apaf- $1 /$ caspase- 9 complex initiates an apoptotic protease cascade. Cell 1997; 91: 479-489.

4. Medema JP, Scaffidi C, Kischkel FC, Shevchenko A, Mann M, Krammer PH et al. FLICE is activated by association with the CD95 death-inducing signaling complex (DISC). EMBO J 1997; 16: 2794-2804.

5. Sprick MR, Rieser E, Stahl H, Grosse-Wilde A, Weigand MA, Walczak H. Caspase-10 is recruited to and activated at the native TRAIL and CD95 death-inducing signalling complexes in a FADD-dependent manner but can not functionally substitute caspase-8. EMBO J 2002; 21: 4520-4530.

6. Luo X, Budihardjo I, Zou H, Slaughter C, Wang X. Bid, a Bcl2 interacting protein, mediates cytochrome $\mathrm{c}$ release from mitochondria in response to activation of cell surface death receptors. Cell 1998; 94: 481-490.

7. Li H, Zhu H, Xu CJ, Yuan J. Cleavage of BID by caspase 8 mediates the mitochondrial damage in the Fas pathway of apoptosis. Cell 1998; 94: 491-501.

8. Stennicke HR, Jurgensmeier JM, Shin H, Deveraux $Q$, Wolf BB, Yang $X$ et al. Pro-caspase-3 is a major physiologic target of caspase-8. J Biol Chem 1998; 273 27084-27090.

9. Talanian RV, Quinlan C, Trautz S, Hackett MC, Mankovich JA, Banach D et al. Substrate specificities of caspase family proteases. J Biol Chem 1997; 272: 9677-9682.

10. Thornberry NA, Rano TA, Peterson EP, Rasper DM, Timkey T, Garcia-Calvo M et al. A combinatorial approach defines specificities of members of the caspase family and granzyme B. Functional relationships established for key mediators of apoptosis. J Biol Chem 1997; 272: 17907-17911.

11. Garcia-Calvo M, Peterson EP, Leiting B, Ruel R, Nicholson DW, Thornberry NA. Inhibition of human caspases by peptide-based and macromolecular inhibitors. J Biol Chem 1998 273: 32608-32613.

12. Berger AB, Witte MD, Denault JB, Sadaghiani AM, Sexton KM, Salvesen GS et al. Identification of early intermediates of caspase activation using selective inhibitors and activity-based probes. Mol Cell 2006; 23: 509-521.

13. Berger $A B$, Sexton KB, Bogyo M. Commonly used caspase inhibitors designed based on substrate specificity profiles lack selectivity. Cell Res 2006; 16: 961-963. 
14. Peri S, Navarro JD, Amanchy $R$, Kristiansen TZ, Jonnalagadda CK, Surendranath V et al. Development of human protein reference database as an initial platform for approaching systems biology in humans. Genome Res 2003; 13: 2363-2371.

15. Stennicke HR, Renatus M, Meldal M, Salvesen GS. Internally quenched fluorescent peptide substrates disclose the subsite preferences of human caspases $1,3,6,7$ and 8 . Biochem J 2000; 350 (Part 2): 563-568.

16. Renatus M, Stennicke HR, Scott FL, Liddington RC, Salvesen GS. Dimer formation drives the activation of the cell death protease caspase 9. Proc Natl Acad Sci USA 2001; 98 14250-14255.

17. Liu X, Kim CN, Yang J, Jemmerson R, Wang X. Induction of apoptotic program in cell-free extracts: requirement for dATP and cytochrome c. Cell 1996; 86: 147-157.

18. Slee EA, Harte MT, Kluck RM, Wolf BB, Casiano CA, Newmeyer DD et al. Ordering the cytochrome c-initiated caspase cascade: hierarchical activation of caspases-2, -3, -6, -7, -8, and -10 in a caspase-9-dependent manner. J Cell Biol 1999; 144: 281-292.

19. Stennicke HR, Deveraux QL, Humke EW, Reed JC, Dixit VM, Salvesen GS Caspase-9 can be activated without proteolytic processing. J Biol Chem 1999; 274 8359-8362.

20. Tu S, McStay GP, Boucher LM, Mak T, Beere HM, Green DR. In situ trapping of activated initiator caspases reveals a role for caspase-2 in heat shock-induced apoptosis. Nat Cell Biol 2006; 8: 72-77.

21. Samraj AK, Keil E, Ueffing N, Schulze-Osthoff K, Schmitz I. Loss of caspase-9 provides genetic evidence for the type I/II concept of CD95-mediated apoptosis. J Biol Chem 2006; 281: 29652-29659.
22. Zheng TS, Hunot S, Kuida K, Momoi T, Srinivasan A, Nicholson DW et al. Deficiency in caspase-9 or caspase-3 induces compensatory caspase activation. Nat Med 2000; 6: 1241-1247.

23. Leonard JR, Klocke BJ, D'Sa C, Flavell RA, Roth KA. Strain-dependent neurodevelopmental abnormalities in caspase-3-deficient mice. J Neuropathol Exp Neurol 2002; 61: 673-677.

24. Lassus $P$, Opitz-Araya $X$, Lazebnik $Y$. Requirement for caspase-2 in stress-induced apoptosis before mitochondrial permeabilization. Science 2002; 297: 1352-1354.

25. Mohr A, Zwacka RM. In situ trapping of initiator caspases reveals intermediate surprises. Cell Biol Int 2006; 31: 526-530.

26. Faleiro L, Kobayashi R, Fearnhead H, Lazebnik Y. Multiple species of CPP32 and Mch2 are the major active caspases present in apoptotic cells. EMBO J 1997; 16: 2271-2281.

27. Stoka V, Turk B, Schendel SL, Kim TH, Cirman T, Snipas SJ et al. Lysosomal protease pathways to apoptosis. Cleavage of bid, not pro-caspases, is the most likely route. $\mathrm{J} \mathrm{Biol}$ Chem 2001; 276: 3149-3157.

28. Pop C, Fitzgerald P, Green DR, Salvesen GS. Role of proteolysis in caspase-8 activation and stabilization. Biochemistry 2007; 46: 4398-4407.

29. Chang DW, Xing Z, Capacio VL, Peter ME, Yang X. Interdimer processing mechanism of procaspase-8 activation. EMBO J 2003; 22: 4132-4142.

30. Golks A, Brenner D, Krammer PH, Lavrik IN. The c-FLIP-NH2 terminus (p22-FLIP) induces NF-kappaB activation. J Exp Med 2006; 203: 1295-1305.

31. Stennicke HR, Salvesen GS. Caspases: preparation and characterization. Methods 1999; 17: $313-319$

32. Stennicke HR, Salvesen GS. Caspase assays. Methods Enzymol 2000; 322: 91-100.

Supplementary Information accompanies the paper on Cell Death and Differentiation website (http://www.nature.com/cdd) 